\title{
Sorting Nexin 6 interacts with Breast Cancer Metastasis Suppressor-1 and promotes transcriptional repression
}

José Rivera $^{{ }^{*}}$, Diego Megías ${ }^{2}$ and Jerónimo Bravo ${ }^{3 *}$

${ }^{1}$ Centro Nacional de Investigaciones Cardiovasculares Carlos III, C/ Melchor Fernández Almagro 3, E-28029 Madrid, Spain. Email: jrivera@cnic.es. Phone: (+34) 91 4531200; Fax:+ (34) 914531245.

${ }^{2}$ Confocal Microscopy Unit, Centro Nacional de Investigaciones Oncológicas, C/ Melchor Fernández Almagro 3, E-28029 Madrid, Spain. Email: dmegias@cnio.es

${ }^{3}$ Instituto de Biomedicina de Valencia (IBV-CSIC), Jaime Roig 11, 46010 Valencia, Spain. Email: jbravo@ibv.csic.es

${ }^{*}$ Corresponding author

Running title: SNX6 interacts with BRMS1and repress transcription

KEY WORDS: Protein-protein interaction, Yeast two-hybrid assay, Binding domain, FRET, BRMS1, SNX6.

Grant sponsor: MCyT; Grant number: SAF2006-10269. Grant sponsor: MICINN; Grant number: SAF2008-04048-E, SAF2009-10667. Grant sponsor: CSIC; Grant number: 200820I020. Grant sponsor: Fundación-Mutua-Madrileña. Grant sponsor: Conselleria de Sanitat, Generalitat valenciana; Grant number: AP001/10.

Total Number of text figures and tables: 4 Figures, 1 Table and 2 Supplemental Figures. 


\section{ABSTRACT}

Sorting nexin 6 (SNX6), a predominantly cytoplasmic protein involved in intracellular trafficking of membrane receptors, was identified as a TGF $\beta$ family interactor. However, apart from being a component of the Retromer, little is known about SNX6 cellular functions. Pim1-dependent SNX6 nuclear translocation has been reported suggesting a putative nuclear role for SNX6. Here we describe a previously nonreported association of SNX6 with Breast Cancer Metastasis Suppressor 1 (BRMS1) protein detected by a yeast two-hybrid screening. The interaction can be reconstituted in vitro and further FRET analysis confirmed the novel interaction. Additionally, we identified their coiled-coil domains as the minimal binding motives required for interaction. Since BRMS1 has been shown to repress transcription, we sought the ability of SNX6 to interfere with this nuclear activity. Using a standard gene-reporter assay, we observed that SNX6 increases BRMS1-dependent transcriptional repression. Moreover, over-expression of SNX6 was capable of diminishing trans-activation in a dosedependent manner. 


\section{Introduction}

Sortin nexins (SNX) are a family of cytoplasmic proteins involved in intracellular trafficking of membrane receptors and characterized by the presence of a Phoxhomology domain (PX) which binds phosphoinositides (Worby and Dixon 2002) and several proteins (Ishibashi, Maita et al. 2001; Parks, Frank et al. 2001; Vollert and Uetz 2004) upstream of a coiled-coil domain (Parks, Frank et al. 2001). Later, the adjacent Cterminal region of PX was defined as a BAR domain (Habermann 2004). SNX6 which was identified as an interactor of the TGF-ß family of receptor serine-threonine kinases (Parks, Frank et al. 2001), is part of the Retromer complex mediating retrograde transport of transmembrane cargo from endosomes to the trans-Golgi. Moreover, overexpression of SNX6 interferes TGF-ß signalling (Parks, Frank et al. 2001), a receptor largely involved in metastasis.

Interaction with Pim-1 oncogene phosphorylates SNX6 leading to its nuclear translocation (Ishibashi, Maita et al. 2001). However, the precise functions of SNX6 within the nuclear compartment have not been reported.

Breast cancer metastasis suppressor-1 (BRMS1), a member of a growing metastasis suppressors family, significantly reduce breast (Seraj, Samant et al. 2000) and melanoma (Shevde, Samant et al. 2002) metastasis without affecting primary tumour growth. Mechanism of action includes restoration of cell-cell communication (Saunders, Seraj et al. 2001), phosphoinositides signalling reduction (DeWald, Torabinejad et al. 2005) and gene repression (Meehan, Samant et al. 2004; Cicek, Fukuyama et al. 2005; Rivera, Megias et al. 2007; Yang, Zhang et al. 2008).

In a search for proteins binding to BRMS1, we identify SNX6, as a novel BRMS1 binding partner. GST-pull-down experiments and FRET analysis validated this interaction both in vitro and in vivo. We studied the interaction in more detail and found 
that the minimal segment of SNX6 sufficient for BRMS1 binding consists of amino acids 300-406, a segment predicted to form a coiled-coil. BRMS1 sequence contains two predicted coiled-coil (CC) motifs and we show that exclusively the N-terminal CC1, recently crystallized (Spinola-Amilibia, Rivera et al. 2008), is implicated in the interaction. In addition, our data suggest that combined over-expression of the newly detected interactors further enhances transcriptional repression in a luciferase reporter assay. Furthermore, the interaction could indicate a potential mechanism by which BRMS1 might regulate intracellular membrane receptor trafficking of TGF- $ß$ receptor family members and thus affecting TGF- $\beta$ signalling pathway which has largely been involved in metastasis. 


\section{MATERIALS AND METHODS.}

\section{Cell culture and transient transfection experiments.}

HeLa, U2OS, T47D and HEK293T cells were cultured in DMEM containing 10\% foetal bovine serum, 2mM glutamine and 1\% penicillin/streptomycin. Melanoma cells were maintained as described (Rivera, Megias et al. 2007). Transfections were performed using Lipofectamine2000 (Invitrogen) following manufacturer's instructions.

\section{Plasmids.}

Human BRMS1 cDNA (Rivera, Megias et al. 2007) was used as a template for PCR and inserted into pAS2.1 (Clontech) to generate pAS2.1-BRMS1 bait vector. Deletion mutants were also created containing: the first putative BRMS1 coiled-coil domain (pAS2.1/CC1; residues 1-88); a BRMS1-CC1 deletion mutant (pAS2.1/ACC1; 89-246); a construct lacking both putative CCs domains (pAS2.1/ACCs; 181-246); and a construct spanning both CCs (pAS2.1/CCs; residues 1-180). Subcloning into pET-28 (Novagen), pCMV-HA and pEYFP-N1 (Clontech) create pET28-BRMS1, pCMV-HABRMS1 and pEYFP-N1-BRMS1 vectors. Entire SNX6 sequence was cloned into pGEX-6-P2 (GE-Healthcare), pECFP-N1, pACT2 and pCMV-Tag2 (Stratagene) rendering pGEX-6-P2/SNX6, pECFP-N1-SNX6, pACT2/SNX6 and pCMV-Tag2SNX6 plasmids. Deletion mutants of SNX6 for yeast system were also created: pACT2/PX (residues 1-180); pACT2/BAR (300-406); pACT2/CC (181-406). Detailed information of specific primers and restriction sites are available upon request.

\section{Yeast two-hybrid.}

A human mammary gland cDNA-library (Clontech) was screened with pAS2.1BRMS1 vector as described (Rivera, Abrams et al. 2007). For sub-mapping interactions, constructs encoding distinct portions of SNX6 and BRMS1 were cloned into pACT2 and pAS2.1 vectors respectively. Sequential transformations by lithium acetate into 
AH109 yeast strain (James, Halladay et al. 1996) produced moderate-fast growing colonies on high-stringency selection media lacking adenine, tryptophan, leucine and histidine after 5-7 days according to the Matchmaker protocol (Clontech). Grown colonies were subjected to a colony-lift filter assay for $\beta$-galactosidase activity. Inserts were identified by DNA sequencing. Chemicals were from Sigma-Aldrich (St. Louis, MO) unless specified.

\section{Glutathione S-transferase pull-down}

GST, GST-SNX6 and 6xHis-BRMS1 proteins were expressed in Escherichia coli BL21 (DE3) cells and proteins were expressed as described (Rivera, Abrams et al. 2007). Purifications of GST or GST-SNX6 were performed using Glutathione Sepharose4B (GE-Healthcare) beads according to the manufacturer's protocols. Equal amounts of GST-fusion proteins, assessed by Coomasie blue staining, were incubated with bacterial lysate containing over-expressed His6-BRMS1 protein in 50mM HEPES, $\mathrm{pH}$ 7.5, 50mM NaCl, 0.1\% NP-40 with proteases inhibitors (Roche) binding buffer. After overnight incubation at $4^{\circ} \mathrm{C}$ glutathione sepharose beads were collected by centrifugation and extensively washed with binding buffer. Proteins were eluted in $2 \mathrm{X}$ SDS buffer fractionated onto 12\% SDS-PAGE and then subjected to Western blot analysis. Nitrocellulose membranes were probed with anti-6xHis antibody (Clontech), before ECL detection (GE-Healthcare).

\section{Confocal microscopy and Fluorescence resonance energy transfer (FRET) analysis}

Cells grown on glass cover-slips were transfected for $48 \mathrm{~h}$, then fixed for $15 \mathrm{~min}$ with $4 \%$ paraformaldehyde, permeabilized with $0.5 \%$ Triton $\mathrm{X}-100$ for $30 \mathrm{~min}$ and blocked in TNB buffer (100mM Tris- $\mathrm{HCl} ; 150 \mathrm{mM} \mathrm{NaCl}$ and 0.5\% Blocking reagent (Boehringer)) for $1 \mathrm{~h}$ at room temperature. Next, cover-slips were sequentially incubated with $\alpha$-HA (BAbCO; HA.11) and $\alpha$-SNX6 (Santa Cruz; K-18) antibodies before 
incubation with secondary antibodies AlexaFluor488 and Cy3-coupled respectively (Jackson Laboratories, West Grove, PA). Images were collected on a Leica-TCS-SP5 laser scanning confocal microscope equipped with a HCX PL APO 63x immersion oil objective (1.4NA). SNX6-ECFP and BRMS1-YFP co-transfected cells were examined

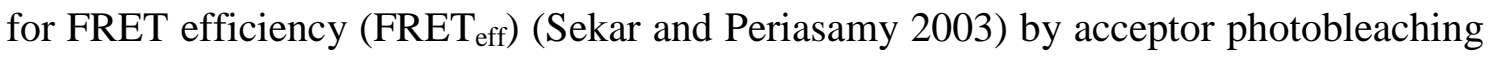
method (Gu, Di et al. 2004). Energy transfer was detected as an increase in donor fluorescence (ECFP) after photobleaching of the acceptor molecules (EYFP). Images were background-corrected and FRET $T_{\text {eff }}$ calculated: FRET $T_{\text {eff }}=\left(D_{\text {post }}-D_{\text {pre }}\right) / D_{\text {post }}$; for all $D_{\text {post }}>D_{\text {pre, where }} D_{\text {pre }}$ and $D_{\text {post }}$ are donor intensities before and after photobleaching, respectively. Polyclonal anti-HDAC2 antibody was from Abcam (ab7029).

\section{Luciferase reporter assay.}

Trans-activation activity assays were performed similarly to previous reports (Meehan, Samant et al. 2004; Hurst, Xie et al. 2008). Briefly, HEK293 and T47D cells were plated in 24-well plates and allowed to attach. After 24h cells were co-transfected, using Lipofectamine2000 in Opti-MEM medium, with various combinations of the following plasmids pCMV-HA/BRMS1 (200 ng), pCMV-Tag2/SNX6 (100; 200 or 250 ng) and pGL-G5 luciferase reporter plasmid, containing five GAL4 binding sites upstream to the E1B promoter driving the expression of firefly luciferase (Zurcher, de la Luna et al. 1996). Assays were conducted in triplicate wells and total DNA amounts were uniformly adjusted with pcDNA3 vector to $0.5 \mu \mathrm{g}$ per well. $48 \mathrm{~h}$ after transfection, cells were lysed, and $20 \mu \mathrm{l}$ of cell lysate were used to measure transactivation activity, using the Dual-Luciferase Reporter system (Promega), in a Glomax luminometer. To allow the normalization of Firefly luciferase readings based on transfection efficiencies, a co-reporter vector expressing Renilla luciferase (pRLSV40) was used as a transfection control at a ratio of 1:20 in the transfection mixture. Relative luciferase activities were 
calculated as the light units relative to the reporter plasmid controls and plotted. Data are expressed as percent luciferase activity relative to empty plasmid transfectants set as $100 \%$. 


\section{RESULTS}

\section{Identification of SNX6 as a BRMS1-interacting protein section}

Identification of new partners can provide useful information about protein functions. A human mammary gland cDNA library was screened by a yeast two-hybrid screen using a bait plasmid encompassing the entire human BRMS1-coding sequence that did not show trans-activation activity by itself upon over-expression and served as a negative control (Table 1). The protein, which is 246 amino-acids long includes two predicted coiled-coil regions, which could account for protein-protein interactions (CC1 and CC2), and recently characterized localization signals, for nuclear export (NES) and nuclear localization (NLS1 and NLS2) (Fig. 1A) (Rivera, Megias et al. 2009).

Screening more than $10^{6}$ yeast transformant colonies for activation of both the His and $L a c Z$ reporter genes yielded several positive clones that grew up on highly selective medium. Two independent clones expressed higher levels of $\beta$-galactosidase ( $\beta$-gal) upon a lift-colony filter assay (Fig. 1B) compared to the remaining clones. After isolation and further plasmid purification of the selected clones, both inserts were different in size, but subsequent sequence analysis revealed that both encoded for human tumour necrosis factor receptor-associated factor 4 (TRAF4)-associated factor 2, also known as Sorting nexin 6 (SNX6) (Parks, Frank et al. 2001). One of them encoded the full length mRNA and was therefore used in subsequent cloning experiments.

SNX6 is composed of a BAR (Bin/Amphiphysin/Rvs) domain at the carboxyterminal end (Habermann 2004) and a Phox-homology (PX) domain at its N-terminal end (Fig. 1A). Interestingly, the second one of the two identified clones encoded for a shorter form lacking the $180 \mathrm{~N}$-terminal residues spanning the PX domain. This domain binds phosphoinositides and has been described also as a protein-protein interaction 
domain (Ishibashi, Maita et al. 2001; Parks, Frank et al. 2001; Vollert and Uetz 2004). Thus, our finding suggests that the PX domain of SNX6 is not involved in BRMS1SNX6 interaction.

\section{BRMS1 interacts with SNX6 in vitro and co-localize in human cells.}

In order to validate the significance of this interaction and determine whether the interaction between SNX6 and BRMS1 was direct or involved additional (yeast) proteins we tested binding in an in vitro assay system. Given that commercial antibodies against BRMS1 showed non reproducible results in our hands for coimmunoprecipating endogenous proteins, we decided to perform more robust approaches over-expressing in a prokaryotic system both interacting proteins, as glutathione-S-transferase (GST) or histidine-tagged fusion proteins respectively in a prokaryotic system. GST pull down experiments using GST/SNX6 fusion protein was mixed with over-expressed 6xHis/BRMS1 full length protein. The GST-SNX6 fusion protein was purified from the mixture using glutathione sepharose4B beads and the pulled down proteins were tested for co-precipitation of the BRMS1 protein by western blotting using an antibody raise against 6xhistidine-tag. As shown in Fig. 1C (lane 3) the full length BRMS1-His tagged protein bound to GST-SNX6 in vitro. A single protein band was specifically recovered in the GST/SNX6 eluate (Fig. 1C, lane 3). This protein, running slower than the expected molecular mass $(32.3 \mathrm{kDa})$ reacted with the anti-6xHis antibody on western blots. Thus, we found that BRMS1 was specifically eluted from the sepharose beads containing GST/SNX6. GST protein used as a control in the assay instead of GST-SNX6, showed no co-precipitation of BRMS1 protein (Fig. 1C, lane 2), indicating that the observed binding is specific for SNX6. The presence of 
GST and GST/SNX6 was specifically confirmed in the same assay using anti-GST antibody (Fig. 1C lanes 5-6). Thus, we conclude that SNX6 and BRMS1 bind each other in vitro and interact in vivo in a yeast assay.

The reported subcellular localisation of SNX6 is mainly cytoplasmic (Parks, Frank et al. 2001; Worby and Dixon 2002) although nuclear localisation has also been shown (Ishibashi, Maita et al. 2001). However, BRMS1 is mostly nuclear although it has also been detected in the cytosol (Rivera, Megias et al. 2007; Stafford, Vaidya et al. 2008). We reasoned that SNX6 and BRMS1 have to exhibit at least partial co-localization if their interaction is physiologically relevant. Therefore, we performed a co-localization analysis. Since we could not reliably detect endogenous BRMS1 protein by commercially available antibodies, we assessed the subcellular localization of HAtagged BRMS1 transiently over-expressed in human cells followed by indirect immunofluorescence detection. We observed a predominantly nuclear localisation pattern of the recombinant BRMS1 proteins upon transient transfection (Fig. 2A-B), in agreement with previous reports (Ishibashi, Maita et al. 2001; Worby and Dixon 2002; Rivera, Megias et al. 2007; Stafford, Vaidya et al. 2008). As observed in transfection experiments, endogenous (Fig. 2A) and over-expressed SNX6 (Fig. 2B) partly colocalised with differently tagged BRMS1. This co-localisation was detected more intensively in the nuclei (Fig. 2A cytofluorogram) of different human cell types (Fig. 2A-B and supplementary Figs. 1 and 2).

\section{Direct SNX6-BRMS1 proteins interaction detected by FRET.}

To complement co-localization studies and further examine the SNX6-BRMS1 interaction we performed a fluorescence resonance energy transfer (FRET) analysis. To 
achieve this goal, full-length BRSM1 and SNX6 proteins were tagged to the N-terminus of enhanced versions of yellow (EYFP) and cyan (ECFP) fluorescence proteins respectively. Both constructs were co-transfected into human cells and FRET efficiency $\left(\mathrm{FRET}_{\text {eff }}\right)$ was measured by acceptor photobleaching method as previously reported (Gu, Di et al. 2004). This method calculates FRET eff $_{\text {as }}$ increments in the emission of the ECFP donor after photobleaching of the EYFP acceptor, which take place exclusively once both fluorophores are in close vicinity within a distance of a few nanometers and it is indicative of protein-protein interaction. Several plasmid pair-wise combinations were co-transfected into mammalian cells as negative controls, including among others co-transfection of pEYFP-N1-BRMS1 or pECFP-N1-SNX6 with a construct encoding for the full length of an unrelated molecule (pECFP-N1-p27 ((Fuster, Gonzalez et al.) and pEYFP-N1-NMI (Rivera et al. unpublished data) respectively) as shown in supplementary Fig. S1. After photobleaching of randomly selected areas with the YFP-light, the ECFP channel increased considerably both in the nucleus and cytoplasm of co-transfected cells (Fig. 2B and C) while no increase was observed in cells co-transfected with the described unrelated molecules, even though some of them (p27-ECFP and BRMS1-EYFP) showed a perfect co-localization into the nuclear compartment (Supplementary Fig. S1). Moreover, the FRET eff $_{\text {values, measured }}$ and averaged from at least ten cells along different experiments, consistently demonstrated, a higher FRET $_{\text {eff }}$ values within the nuclear compartment $(44 \pm 11.5 \%)$ compare to those observed for the cytosol (27.5 $\pm 7.5 \%)$. Interestingly, these differences showed statistical significance $(\mathrm{P}<0.05)$, although we do not know the biological significance yet (Fig. 2C).

FRET $_{\text {eff }}$ was also measured by the alternative acceptor sensitized emission method (see supplementary material and methods). As shown in supplementary Fig. S2, cells 
co-transfected with constructs encoding ECFP-SNX6 and EYFP-BRMS1 fusion proteins confirmed the acceptor photobleaching FRET $_{\text {eff }}$ values reported above. Negative controls were also run in parallel (Supplementary Fig. S2).

Thus, FRET analysis confirms that interaction occurs, providing strong evidence for the association of SNX6 with BRMS1 in both cytoplasmic and nuclear compartments and thus corroborating the results of in vivo yeast two-hybrid, pull-down assays, and co-localization studies. Our findings suggest that SNX6 may play a role in the nucleus. Similarly, cytosolic presence of BRMS1 does not seem to be an artefact and might play a functional role in that compartment.

The PX motif was previously described as a binding site for diverse protein partners (Ishibashi, Maita et al. 2001; Xu, Seet et al. 2001; Worby and Dixon 2002). Since this motif is absent in one of the two fished out clones upon yeast screen, we hypothesized that this motif is not essential for the interaction. To test this hypothesis we engineered and over-expressed a deletion mutant of SNX6 where the PX domain

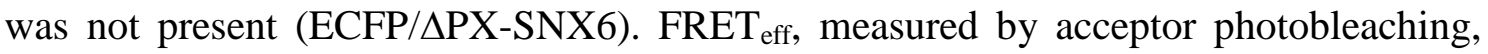
within the nucleus and cytoplasm showed values of $36.5 \pm 7.5 \%$ and $33.6 \pm 8 \%$ respectively (Fig. 2C), which were indeed similar to those obtained upon co-transfection of full-length proteins, confirming that PX domain from SNX6 is dispensable for its interaction with BRMS1.

\section{Identification of minimal domains involved in BRMS1- SNX6 interaction}

In order to further map the regions of SNX6 and BRMS1 proteins that are relevant for its interaction, we created a series of truncated constructs (Fig. 3A-B) and perform yeast two-hybrid experiments. Engineered SNX6 constructs-domains were back- 
transformed into yeast together with the various BRMS1 constructs. After assessing self-activation inability for all the designed constructs (data not shown), all possible pair-wise combinations were tested for positive interactions by the activation of both reporter genes.

We did not detect any binding of deletion mutant expressing solely the SNX6-PX domain (PX 1-180) with any of the BRMS1 constructs assayed, as judged by the inability of yeast cells to grow on deficient media (Table 1). This result reinforces the hypothesis that the PX domain is dispensable for the interaction with BRMS1. Instead, SNX6 full length construct strongly activated both reporter genes in yeast cells coexpressing all three constructs where the first $88 \mathrm{~N}$-terminal residues of BRMS1 were present (FL, CC1 and CCs). A series of further truncated constructs mapped the minimal SNX6-binding site within BRMS1 protein to amino acids 1-88, containing the predicted coiled-coil domain (Fig. 3C and Table 1), thus highlighting its crucial role. We therefore suggest that the N-terminal fragment of BRMS1, harbouring a predicted coiled-coil domain, is necessary and sufficient for binding to SNX6.

Results on Table 1 show that a construct encompassing the entire BAR domain of SNX6 achieved interaction to the same extent that a shorter construct including a coiled-coil region, concluding that the C-terminal end of SNX6, shorter than the BAR domain, is necessary and sufficient for binding to BRMS1.

Of note is the observation that BRMS1-CC1 construct showed the strongest $\beta$-gal activity. This result suggests that a negative regulatory effect might exist in the context of full length BRMS1 (e.g., the CC1 region might have been partially shielded in the presence of BRMS1 C-terminal end). 
SNX6 affects trans-activation and contributes to BRMS1-transcriptional repression

The results shown above indicate that BRMS1 interacts with SNX6 both in the nucleus and the cytoplasm. It has previously been reported that BRMS1 affects transcriptional repression by its interaction with different components of the mSin3 HDAC complex (Meehan, Samant et al. 2004). We sought to investigate a possible functional relationship between BRMS1 and SNX6 by testing whether SNX6 might play a role in the modulation of transcriptional repression. To address this hypothesis we carried out a conventional trans-activation assay as previously described (Meehan, Samant et al. 2004). We performed an standard luciferase reporter assay in human cells transfected with a construct (pGL-G5) containing five binding sites of Gal4 promoter (Zurcher, de la Luna et al. 1996). Proliferating HEK 293T cells exhibited a basal reporter activity that we set as $100 \%$ activity (Fig. 4A-B). As expected, trans-activation activity was specifically repressed to $65 \%$ upon the transient expression of BRMS1 (Fig. 4A). Importantly, co-expression of BRMS1 and SNX6 exhibited an additive effect on transcriptional repression reducing reporter activity up to more than twofold in a SNX6-dose dependent manner (Fig. 4A). The combination of BRMS1 expression with increasing amounts of SNX6 constructs displayed a statistically significant $(\mathrm{P}<0.001)$ increase in transcriptional repression capability as compared to that exerted by BRMS1 when transfected alone (Fig. 4A). Moreover, transient expression of increasing amounts of SNX6 transfected plasmid also reduced luciferase reporter activity almost as effectively as BRMS1 when the highest SNX6 plasmid dose was used (Fig. 4B). Interestingly, the latter result suggests that SNX6 might play a role in transcriptional repression, although, no trans-activation domain has been reported for this protein. 
Whether this effect can be mediated by SNX6 on its own or by acting as a co-repressor is not known yet and should be further analysed.

Similar and consistent repression of a reporter luciferase activity was also achieved when a different human T47D breast cell line was used (data not shown), suggesting that the observed effect on transcriptional repression might not be cell specific but a general mechanism.

Altogether, these combined results hypothesize a conceivable mechanism by which SNX6 and BRMS1 could modulate gene expression in mammalian cells.

\section{DISCUSSION}

In the present study we demonstrate that SNX6 and BRMS1 interact in vivo in a yeast two-hybrid system. Some previously reported BRMS1-interacting proteins, were also fished out in our assay (data not shown), assessing the consistency for the performance of our screening.

This interaction can be reconstituted in vitro indicating that no additional yeast proteins are involved. Glutathione-S-Transferase (GST) pull-down assays demonstrated that an over-expressed construct encompassing the entire sequence of BRMS1 precipitated SNX6, validating yeast results and suggesting direct binding. The proteinprotein interaction was further confirmed by FRET analysis. As shown in Fig. 2 and Supplementary Fig. 1 and 2, mammalian cells over-expressing ECFP-SNX6 and EYFPBRMS1 showed specific and considerably high FRET $_{\text {eff }}$ values, demonstrating that SNX6-BRMS1 interaction occurs in vivo. The identification of this novel interaction of BRMS1 with a bona fide cytosolic protein is in agreement with recent reports 
demonstrating the nucleo-cytoplasmic shuttling capacity of BRMS1 and its cytosolic presence (Stafford, Vaidya et al. 2008; Rivera, Megias et al. 2009).

Moreover, the interaction of BRMS1 with SNX6 reported in this work opens up the possibility of a cytosolic role for this suppressor of breast cancer metastasis.

A previous report, using oligonucleotide microarrays coupled to a robust platform of informatic analysis, have shown that in response to BRMS1 re-expression the signature of diverse secretory/trafficking pathway-genes changed significantly. Interestingly, SNX1 and SNX5, two other members of the sortin nexin family, were repressed upon BRMS1 re-expression (Champine, Michaelson et al. 2007).

A common feature of the SNX family is its PX domain. SNX6 contains a predicted coiled-coil region C-terminal to the PX domain. Whereas homo and heteromeric interactions with other members of the family involve both PX and coiledcoil domains, the majority of the interactions with membrane receptors, as well as with the oncogene Pim-1, take place by the PX motif (Ishibashi, Maita et al. 2001; Xu, Seet et al. 2001; Worby and Dixon 2002). Interestingly, one of the two cDNAs pulled out in our yeast two-hybrid screen lacked this motif, suggesting that, unlike most of the interactions reported for SNX6, the PX domain is dispensable for its interaction with BRMS1. This fact was confirmed in human cells by FRET analysis where proteinprotein interaction efficiency, in cells transfected with a PX deletion mutant $(\Delta \mathrm{PX}$ SNX6/ECFP), was indistinguishable from cells over-expressing full-length SNX6. In addition, we have precisely mapped the regions mediating the interaction to the predicted coiled-coil motif of SNX6 and the CC1-BRMS1 located at the N-terminal end.

It is worth to mention that we have observed that SNX6 and BRMS1 interact both in the cytoplasm and the nucleus. Despite the fact that no functional activity had been 
reported for SNX6 in the nucleus, it has been shown that Pim-1-mediated phosphorylation of SNX6 leads to its translocation from cytoplasm to the nucleus (Ishibashi, Maita et al. 2001). Moreover, several PX domain-containing proteins interact with nuclear proteins (Vollert and Uetz 2004) relating lipids with nuclear functions (Shi and Gozani 2005). BRMS1 has largely been restricted to the cell nucleus (Samant, Seraj et al. 2000) where it acts as a transcriptional co-repressor by interacting with diverse components within the large mSin3-HDAC complex or in a smaller complex with HDAC1 (Meehan, Samant et al. 2004).

We therefore sought to address whether the novel SNX6-BRMS1 interaction colocalise with the HDAC complex. Despite the fact that FRET protein-protein interaction could not be detected between SNX6-ECFP and the endogenous HDAC2, likely due to the large size of the specific antibody anti-HDAC used, co-localization studies by confocal microscopy showed that BRMS1-SNX6 complex (Fig. 2B-C) co-localizes with the endogenous HDAC complex upon transient expression (Fig. 2D). These results raise the hypothesis of BRMS1-SNX6 playing a role in transcription. Furthermore, using a well established luciferase reporter assay, we show that over-expression of SNX6 in human cells is capable of modulating the transcriptional machinery revealing a previously non-described function for SNX6 within the nuclear compartment and confirming that our findings might be of physiological relevance.

The absence of a trans-activation domain within the SNX6 sequence suggests that it might act as a transcriptional co-factor. In fact, a previous report, using a similar reporter assay, clearly indicated that SNX6 interferes with TGF- $\beta$ signalling in a dose dependent manner, although the assay did not provide a direct measure for any single component of the pathway but the ability of TGF- $\beta$ to induce gene expression, concluding that the increasing amounts of SNX6 modified the TGF- $\beta$ receptors. 
Importantly, although the significance of the BRMS1-SNX6 interaction is still not fully understood, the co-expression, in different human cell types, of BRMS1 and SNX6 in a gene reporter assay enhanced the transcriptional repression exerted by BRMS1, as judged by the reduction of luciferase trans-activation (Fig. 4). The transcriptional blockade achieved after co-expression although modest, it was consistently observed along five different experiments performed in triplicate and found statistically significant. Further studies are needed to evaluate the biological significance of the observed transcriptional repression.

We show that SNX6 affects transcriptional repression. This observation might be in agreement with previous data since SNX6 was first identified as a Smad1 protein partner (Kim, Wang et al. 2000) and later on reported to interact to a different extent with several members of the TGF- $\beta$ family of serine-threonine kinase receptors interfering with TGF- $\beta$ signalling (Parks, Frank et al. 2001). Interestingly, TGF- $\beta$ signalling have been largely associated to metastasis (Padua and Massague 2009) .

Moreover, it has recently been reported that the interaction of SNX6 with GIT1 enhances degradation of the epidermal growth factor receptor (EGFR) and alters EGFR signalling (Cavet, Pang et al. 2008). EGFR has long been associated to carcinogenesis and it has been proposed that EGFR contributes to intravasation and metastasis (Xue, Wyckoff et al. 2006). The fact that SNX6 directly affects two signalling pathways involved in metastasis provides an interesting functional relationship to our reported molecular interaction between SNX6 and BRMS1.

BRMS1 was first identified as a largely down-regulated gene in highly metastatic breast carcinoma and melanoma cells. Reintroduction of BRMS1 in both human and mouse derived cells correlated with reduced metastatic potential in in vivo metastasis assays (Samant, Seraj et al. 2000; Samant, Debies et al. 2002; Shevde, Samant et al. 
2002). Interestingly, the aggressiveness of the highly metastatic C8161 melanoma cells and MDA-MB-231 and Hs 578T breast carcinoma cell lines has been recently correlated (Topczewska, Postovit et al. 2006) with the over-expression of a secreted Nodal protein, which is precisely a ligand of the Activin and TGF- $\beta$ family of receptors which interact with SNX6.

In summary, our study provides evidences of a novel interaction between the intracellular trafficking protein SNX6 and the breast cancer metastasis suppressor BRMS1, which is mediated by their respective coiled-coils motives. Furthermore, our results supply for the first time evidences that SNX6 might play a novel role as a modulator of the transcriptional repression machinery. Whether SNX6 is a component of the previously described BRMS1-HDAC complex or constitutes a novel level of transcription regulation is still unknown and should be the focus of further studies.

\section{Acknowledgements}

This study was supported by Grant SAF2006-10269 from the MCyT, Grants SAF2008-04048-E, SAF2009-10667 from MICINN, grant 2008201020 from CSIC, Fundación-Mutua-Madrileña and grant AP001/10 from Conselleria de Sanitat, Generalitat Valenciana. 


\section{REFERENCES}

Cavet, M. E., J. Pang, et al. (2008). "An epidermal growth factor (EGF) -dependent interaction between GIT1 and sorting nexin 6 promotes degradation of the EGF receptor." FASEB J 22(10): 3607-16.

Cicek, M., R. Fukuyama, et al. (2005). "Breast cancer metastasis suppressor 1 inhibits gene expression by targeting nuclear factor-kappaB activity." Cancer Res 65(9): 3586-95.

Champine, P. J., J. Michaelson, et al. (2007). "Microarray analysis reveals potential mechanisms of BRMS1-mediated metastasis suppression." Clin Exp Metastasis 24(7): 551-65.

DeWald, D. B., J. Torabinejad, et al. (2005). "Metastasis suppression by breast cancer metastasis suppressor 1 involves reduction of phosphoinositide signaling in MDA-MB-435 breast carcinoma cells." Cancer Res 65(3): 713-7.

Fuster, J. J., J. M. Gonzalez, et al. "Tumor suppressor p27Kip1 undergoes endolysosomal degradation through its interaction with sorting nexin 6." $\underline{\text { FASEB }}$ J.

Gu, Y., W. L. Di, et al. (2004). "Quantitative fluorescence resonance energy transfer (FRET) measurement with acceptor photobleaching and spectral unmixing." $\underline{\mathrm{J}}$ Microsc 215(Pt 2): 162-73.

Habermann, B. (2004). "The BAR-domain family of proteins: a case of bending and binding?" EMBO Rep 5(3): 250-5.

Hurst, D. R., Y. Xie, et al. (2008). "Alterations of BRMS1-ARID4A interaction modify gene expression but still suppress metastasis in human breast cancer cells." $\underline{\mathrm{J}}$ Biol Chem 283(12): 7438-44.

Ishibashi, Y., H. Maita, et al. (2001). "Pim-1 translocates sorting nexin 6/TRAF4associated factor 2 from cytoplasm to nucleus." FEBS Lett 506(1): 33-8.

James, P., J. Halladay, et al. (1996). "Genomic libraries and a host strain designed for highly efficient two-hybrid selection in yeast." Genetics 144(4): 1425-36.

Kim, R. H., D. Wang, et al. (2000). "A novel smad nuclear interacting protein, SNIP1, suppresses p300-dependent TGF-beta signal transduction." Genes Dev 14(13): 1605-16.

Meehan, W. J., R. S. Samant, et al. (2004). "Breast cancer metastasis suppressor 1 (BRMS1) forms complexes with retinoblastoma-binding protein 1 (RBP1) and the mSin3 histone deacetylase complex and represses transcription." $\underline{\mathrm{J} \text { Biol }}$ Chem 279(2): 1562-9.

Padua, D. and J. Massague (2009). "Roles of TGFbeta in metastasis." Cell Res 19(1): 89-102.

Parks, W. T., D. B. Frank, et al. (2001). "Sorting nexin 6, a novel SNX, interacts with the transforming growth factor-beta family of receptor serine-threonine kinases." J Biol Chem 276(22): 19332-9.

Rivera, J., C. Abrams, et al. (2007). "The MyD116 African swine fever virus homologue interacts with the catalytic subunit of protein phosphatase 1 and activates its phosphatase activity." J Virol 81(6): 2923-9.

Rivera, J., D. Megias, et al. (2007). "Proteomics-based strategy to delineate the molecular mechanisms of the metastasis suppressor gene BRMS1." J Proteome Res 6(10): 4006-18.

Rivera, J., D. Megias, et al. (2009). "Identification of essential sequences for cellular localization in BRMS1 metastasis suppressor." PLoS One 4(7): e6433. 
Samant, R. S., M. T. Debies, et al. (2002). "Identification and characterization of the murine ortholog (brms1) of breast-cancer metastasis suppressor 1 (BRMS1)." Int J Cancer 97(1): 15-20.

Samant, R. S., M. J. Seraj, et al. (2000). "Analysis of mechanisms underlying BRMS1 suppression of metastasis." Clin Exp Metastasis 18(8): 683-93.

Saunders, M. M., M. J. Seraj, et al. (2001). "Breast cancer metastatic potential correlates with a breakdown in homospecific and heterospecific gap junctional intercellular communication." Cancer Res 61(5): 1765-7.

Sekar, R. B. and A. Periasamy (2003). "Fluorescence resonance energy transfer (FRET) microscopy imaging of live cell protein localizations." J Cell Biol 160(5): 62933.

Seraj, M. J., R. S. Samant, et al. (2000). "Functional evidence for a novel human breast carcinoma metastasis suppressor, BRMS1, encoded at chromosome 11q13." Cancer Res 60(11): 2764-9.

Shevde, L. A., R. S. Samant, et al. (2002). "Suppression of human melanoma metastasis by the metastasis suppressor gene, BRMS1." Exp Cell Res 273(2): 229-39.

Shi, X. and O. Gozani (2005). "The fellowships of the INGs." J Cell Biochem 96(6): 1127-36.

Spinola-Amilibia, M., J. Rivera, et al. (2008). "Crystallization and preliminary X-ray diffraction analysis of a breast cancer metastasis suppressor 1 predicted coiledcoil region." Acta Crystallogr Sect F Struct Biol Cryst Commun 64(Pt 12): 1156-8.

Stafford, L. J., K. S. Vaidya, et al. (2008). "Metastasis suppressors genes in cancer." Int J Biochem Cell Biol 40(5): 874-91.

Topczewska, J. M., L. M. Postovit, et al. (2006). "Embryonic and tumorigenic pathways converge via Nodal signaling: role in melanoma aggressiveness." 12(8): 925-32.

Vollert, C. S. and P. Uetz (2004). "The phox homology (PX) domain protein interaction network in yeast." Mol Cell Proteomics 3(11): 1053-64.

Worby, C. A. and J. E. Dixon (2002). "Sorting out the cellular functions of sorting nexins." Nat Rev Mol Cell Biol 3(12): 919-31.

Wouters, F. S., P. J. Verveer, et al. (2001). "Imaging biochemistry inside cells." Trends Cell Biol 11(5): 203-11.

Xu, Y., L. F. Seet, et al. (2001). "The Phox homology (PX) domain, a new player in phosphoinositide signalling." Biochem J 360(Pt 3): 513-30.

Xue, C., J. Wyckoff, et al. (2006). "Epidermal growth factor receptor overexpression results in increased tumor cell motility in vivo coordinately with enhanced intravasation and metastasis." Cancer Res 66(1): 192-7.

Yang, J., B. Zhang, et al. (2008). "Breast cancer metastasis suppressor 1 inhibits SDF1alpha-induced migration of non-small cell lung cancer by decreasing CXCR4 expression." Cancer Lett 269(1): 46-56.

Zacharias, D. A. (2002). "Sticky caveats in an otherwise glowing report: oligomerizing fluorescent proteins and their use in cell biology." Sci STKE 2002(131): pe23.

Zurcher, T., S. de la Luna, et al. (1996). "Mutational analysis of the influenza virus A/Victoria/3/75 PA protein: studies of interaction with PB1 protein and identification of a dominant negative mutant." J Gen Virol 77 ( Pt 8): 1745-9. 


\section{Figure Legends}

Figure 1. In vitro association between SNX6 and BRMS1. (A) Schematic representation of BRMS1 and SNX6 structures displaying boundaries of different domains: CC; Coiled-coil, PX; Phox-Homology. (B) $\beta$-Galactosidase activity of grown co-transformant yeast colonies encoding SNX6 (isolated clones) after two-hybrid assay using BRMS1 bait vector to screen a human breast cDNA library. Positive (+ve) and negative (-ve) controls are also shown as described in the main text. (C) GST or GSTSNX6 proteins were mixed with bacterial cell lysates containing 6xHis-tagged BRMS1 (see Materials and Methods) and pulled-down with Glutathione Sepharose 4B beads. Co-immunoprecipitated proteins were eluted with 2 X Laemli buffer, analyzed on $12 \%$ SDS-PAGE gels and blotted with specific antibodies raised against 6xHis (lanes 1 to 3) or GST (lanes 4 to 6) antibodies. BRMS1 was elicited as a pulled-down protein complex with a GST-SNX6 beads (lanes 3 and 6) but not in the control assays using GST alone (lanes 2 and 5). 6xHis-BRMS1 (lanes 1 and 4) containing samples were shown using $\alpha$-His antibody but not detected with $\alpha$-GST antibody. Protein markers are shown in kDa. 
Figure 2. Co-localization and FRET Detection of SNX6-BRMS1 Interaction. (A) Partial co-localization of SNX6 and BRMS1. HeLa cells were stained using $\alpha$-HA (green colour, middle panel) and anti-SNX6 (red colour, left panel). Right panel shows co-localization represented by a merge of adjacent spots populations overlapping red and green signals. Staining was analyzed by confocal microscopy and images show scanning of a single plane. (B) Representative images of donor (ECFP) and acceptor (EYFP) fluorescence intensity before and after EYFP photobleaching. Images of cotransfected cells with full length BRMS1 acceptor molecule and full length SNX6 (top panels) or a PX-deletion mutants (bottom panels) donor molecule are shown. Scale bar, $20 \mu \mathrm{m} . \mathrm{FRET}_{\text {eff }}(\%)$ measured in randomly selected areas (white boxes) is shown the right-hand panel as pseudo-coloured images relatives to amplitude of $\mathrm{FRET}_{\text {eff }}$ as shown

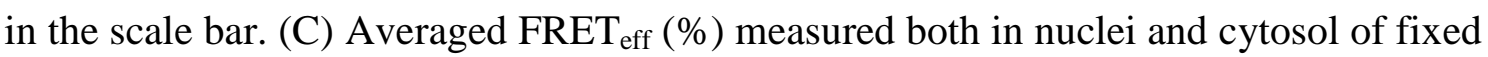
melanoma cells ( $\mathrm{n} \geq 20$ cells for each experiment) as the increase of acceptor molecules following acceptor (BRMS1-EYFP) photo-bleaching. Note significant reduction (* $P<0.05)$ of FRET $_{\text {eff }}$ in cytosol compare to the nucleus in full length transfected proteins. Error bars represent mean \pm SEM from four different independent experiments carried out in duplicates. (D) Representative images of co-transfected cells as described for subpanel (B), where endogenous HDAC-2 was detected by a polyclonal antibody. Pairwise combinations of merged images are shown in the right-hand panel as stated. 
Figure 3. Minimal domains responsible for SNX6-BRMS1 interaction. Diagram of BRMS1 (A) and SNX6 (B) deletion mutants are schematically depicted and referenced to the full length protein sequences. (C) Representative images of yeast cells cotransformed with a prey vector encoding the entire SNX6 and a combination of bait plasmids encoding the complete sequence of BRMS1 (1); 1-88 N-terminal residues containing the first putative coiled-coil domain (2), or both coiled-coil domains (3), or mutants lacking the first (4) or both coiled-coils (5). Co-transformed yeasts were grown on selective medium (SD-T-L) to assess co-transformation and further screened for a Histidine reporter gene (SD-T-L-H). 
Figure 4. Modulation of BRMS1-dependent transactivation by SNX6. T47D transiently transfected cells with 100 ng of pGL-G5 luciferase reporter construct alone (lane 1) or in combination with HA-BRMS1 $(0.20 \mu \mathrm{g})$ (lanes 5 to 8 ) were assayed in a dual-luciferase reporter system. Increasing amounts (0.1; 0.2 or $0.25 \mu \mathrm{g})$ of Flag-SNX6 expression plasmids were used as indicated. Equal amounts of plasmid DNA (0.5 $\mu \mathrm{g} /$ well) was accomplished with pcDNA3 vector. Cells in 24 well plates were lysed in $100 \mu \mathrm{l}$ of passive lysis buffer $48 \mathrm{~h}$ post transfection and $20 \mu \mathrm{l}$ were used for the assay. Luciferase activity was normalized to Renilla measurements to take into account transfection efficiency variations. Data from five independent experiments assayed in triplicate are presented as the mean percentage \pm SD compared to relative luciferase activity of cells transfected only with pcDNA3 vector (lane 1) considered as $100 \%$ activity. * represents statistical significance with $\mathrm{P}<0.05$. 
Table 1. Minimal binding domains involved in the interaction of SNX6 with BRMS1. Different domains of BRMS1 were fused to the Gal4-DNA-binding domain (Gal4-BD). SNX6 fragments were fused to the Gal4 transcription activation domain (Gal4-AD). Protein-protein interaction results in activation of reporter genes allowing yeast cells to grow in synthetic drop-out medium lacking, Tryptophan, Leucine and Histidine (SD/-T/-L/-H). The AH109 yeast cell co-transformed with: pACT2 empty vector and BRMS1; and a pair of interacting proteins previously reported (Rivera, Abrams et al. 2007) were used as negative and positive controls respectively. $\beta$ Galactosidase activity was evaluated by lift-colony filter assay. Growth of cotransformed yeast cells onto SD/-T/-L are shown in brackets. Numbers shown in brackets within the constructs columns represent the spanning residues according to the full length protein as shown in Fig. 3A-B. Intensity of the blue colour is a measure for the interaction strength that was defined as: +++ strong, ++ moderate, + weak, - not detectable. 


\section{Supplementary Information}

\section{Supplementary Figure 1- Basal energy transfer for acceptor photobleaching FRET measurements.}

Representative pictures of donor (ECFP) and acceptor (EYFP) fluorescence intensities before (pre-bleaching columns) and after (post-bleaching columns) EYFP acceptor photobleaching. Images of U2OS co-transfected cells with the indicated full length acceptor (BRMS1/EYFP and NMI/EYFP) and donor molecules (p27/ECFP and SNX6/ECFP), as well as a combination of test proteins (SNX6/ECFP and BRMS1/EYFP) with their respective phluorophore pairs (EYFP and ECFP empty vectors), served as internal negative control for FRET efficiency measurements. FRET $_{\text {eff }}(\%)$ measured in randomly selected areas (white squared boxes) within the nucleus is shown at the most right-hand panel in a pseudo-coloured image relatives to amplitude of FRET eff. $_{\text {. }}$

\section{Supplementary Figure 2- FRET interaction between SNX6/CFP and BRMS1/YFP fusion proteins by sensitized emission technique.}

Protein-protein interaction between SNX6/ECFP and BRMS1/EYFP fusion proteins was carried out by the FRET technique sensitized emission and confocal microscopy. The top panel right column shows areas indicative of interaction between SNX6 and BRMS1 proteins (37\%). FRET efficiency values are indicated and coded as pseudocolour. White line, surrounding the nucleus of U2OS cells co-transfected with BRMS/EYFP and ECFP empty vectors, represents the area of measurement because it showed comparable expression levels to the fluorescence signal observed in control samples. Co-transfection of p27/ECFP (non-interactor partner) and BRMS/EYFP 
represents a negative control for the specificity of the interaction (bottom panel). Low FRET efficiency, in cells co-transfected with the empty control vector pECFP-N1 (ECFP) and pEYFP-N1 (EYFP) or a construct encoding for the full length BRMS1(BRMS/EYFP) due to non specific hydrophobic interaction between the fluorophores are also shown (see Supplementary Material and Methods).

\section{Supplementary MATERIALS AND METHODS.}

\section{Fluorescence Resonance Energy Transfer (FRET) Protein-Protein Interaction Analysis by sensitized FRET technique.}

Interaction analysis between SNX6 and BRMS1 obtained by acceptor photobleaching FRET method was also confirmed by sensitized emission FRET technique. Sensitized emission measurements were performed using the commercially available algorithm incorporated into the Leica SP2 AOBS confocal software. The system equipped with four photomultipliers, 458nm laser for ECFP excitation, a 514 for EYFP excitation and the inverse microscope DMIRE2 were used. Image acquisition was performed with a $63 x / 1.40$ oil objective (Leica). The calculation of the sensitized-acceptor emission is based on images of the donor and acceptor fluorescence acquired from the FRET double-labeled sample, which are corrected for leak-through and cross-excitation using correction factors obtained from the control donor and acceptor single-labeled samples following a method previously described (Wouters, Verveer et al. 2001).

The CFP and YFP signals, accounting for SNX6 and BRMS1 constructs respectively, after excitation with the $458 \mathrm{~nm}$ and 514 lasers was collected between 470-490nm (intensity of the donor) and 530-585 nm (intensity of the acceptor). The intensity of the 
FRET signal was collected by a photomultiplier between $530-585 \mathrm{~nm}$ with the $514 \mathrm{~nm}$ laser.

FRET efficiency (E) value was calculated according to the following equation:

$$
E=\frac{I_{\lambda D}^{A}-b \cdot I_{\lambda D}^{D}-(c-a \cdot b) \cdot I_{\lambda A}^{A}}{I_{\lambda A}^{A}}
$$

Where: $I_{\lambda D}^{A}, I_{\lambda D}^{D}, I_{\lambda A}^{A}$ are fluorescence intensities determined from images from the FRET, double-labeled sample and a, b, and c are correction factors obtained from control donor and acceptor single-labeled sample, where:

$I_{\lambda D}^{A} \quad$ is the acceptor uncorrected fluorescence emission excited by donor light

$I_{\lambda D}^{D} \quad$ is the donor uncorrected fluorescence emission excited by donor light

$I_{\lambda A}^{A} \quad$ is the acceptor uncorrected fluorescence emission excited by acceptor light

a is obtained from an acceptor-only sample by dividing $I_{\lambda D \mathrm{by}}^{D} I_{\lambda A}^{A}$

b $\quad$ is obtained from a donor-only sample by dividing $I_{\lambda D \mathrm{by}}^{\mathrm{A}} I_{\lambda D}^{D}$

c $\quad$ is obtained from an acceptor-only sample by dividing $I_{\lambda D \text { by }}^{A} I_{\lambda A}^{A}$.

Tendency to form dimers via hydrophobic interaction has been reported for GFP and their ECFP and EYFP derivatives, especially at high concentrations (Zacharias 2002). It defines the biochemical limit of detection of signal due to molecular interaction. To determine the detection limit of our analysis mammalian cells were co-transfected with the empty control vectors pECFP-N1 and pEYFP-N1 (Clontech) among other combinations (Supplementary Fig. 2S). 